\title{
The Distribution and Cosmic Density of Relativistic Iron Lines in Active Galactic Nuclei
}

\author{
D. R. Ballantyne \\ Center for Relativistic Astrophysics, School of Physics, Georgia Institute of Technology, \\ Atlanta, GA 30332 \\ david.ballantyne@physics.gatech.edu
}

\begin{abstract}
X-ray observations of several active galactic nuclei show prominent iron Kshell fluorescence lines that are sculpted due to special and general relativistic effects. These observations are important because they probe the space-time geometry close to distant black holes. However, the intrinsic distribution of Fe line strengths in the cosmos has never been determined. This uncertainty has contributed to the controversy surrounding the relativistic interpretation of the emission feature. Now, by making use of the latest multi-wavelength data, we show theoretical predictions of the cosmic density of relativistic Fe lines as a function of their equivalent width and line flux. We are able to show unequivocally that the most common relativistic iron lines in the universe will be produced by neutral iron fluorescence in Seyfert galaxies and have equivalent widths $<100 \mathrm{eV}$. Thus, the handful of very intense lines that have been discovered are just the bright end of a distribution of line strengths. In addition to validating the current observations, the predicted distributions can be used for planning future surveys of relativistic Fe lines. Finally, the predicted sky density of equivalent widths indicate that the X-ray source in AGNs can not, on average, lie on the axis of the black hole.
\end{abstract}

Subject headings: accretion, accretion disks — galaxies: active — galaxies: nuclei - galaxies: Seyfert — X-rays: galaxies

\section{Introduction}

The X-ray spectrum of many active galactic nuclei (AGNs) exhibits a strong emission feature at an energy of $6.4 \mathrm{keV}$ that is due to the fluorescence of weakly ionized iron in a dense 
and relatively cold medium (e.g., Nandra et al. 1989; Pounds et al. 1990; Nandra \& Pounds 1994). The strength of the emission feature requires that the region responsible for the fluorescence subtend about half the sky as seen from the illuminating X-ray source (e.g., George \& Fabian 1991). As the variability properties of AGN place the X-ray source within 10 Schwarzschild radii from the black hole (e.g., Grandi et al. 1992; Uttlev 2007), it is likely that relativistic effects may alter the observed line shape (Fabian et al. 1989; Laor 1991) allowing it to be a powerful probe of space-time curvature. Several examples of relativistically broadened $\mathrm{Fe} \mathrm{K} \alpha$ lines have been detected in the spectra of AGNs over the last decade (e.g., Tanaka et al. 1995; Fabian et al. 2002b; Reynolds \& Nowak 2003; Miller 2007; Fabian et al. 2009). These detections are leading to measurements of black hole spins and accretion disk dynamics (Brenneman \& Reynolds 2006; Reynolds \& Fabian 2008).

The strength of the Fe line emission depends on only four parameters: (i) the shape of the illuminating continuum (Ballantyne et al. 2002; Ballantyne \& Ross 2002), which in AGNs is a power-law with photon index $\Gamma$; (ii) the abundance of iron (relative to the solar value) in the accretion disk (Ballantvne et al. 2002), $A_{\mathrm{Fe}}$; (iii) the ionization parameter of the illuminated disk (Ballantyne et al. 2002), $\xi=4 \pi F_{X} / n_{\mathrm{H}}$, where $F_{X}$ is the illuminating flux, and $n_{\mathrm{H}}$ is the density of the illuminated slab; and (iv) the reflection fraction $R$, a measure of the relative strength of the reflection component in the observed spectrum (this is related to the covering factor of the accretion disk). Recently, multi-wavelength survey data has been able to measure correlations between each of the first three of these parameters to $\lambda=L_{\mathrm{bol}} / L_{\mathrm{Edd}}$, the Eddington ratio of AGNs (Risaliti et al. 2009; Inoue et al. 2007; Netzer \& Trakhtenbrot 2007). Here, $L_{\text {bol }}$ is the bolometric luminosity of an AGN, and $L_{\mathrm{Edd}}=1.3 \times 10^{38}\left(M_{\mathrm{BH}} / M_{\odot}\right) \mathrm{erg} \mathrm{s}^{-1}$ is the Eddington luminosity of a black hole with mass $M_{\mathrm{BH}}$ and $M_{\odot}$ is the mass of the Sun. In addition, various surveys have been able to measure the black hole mass distribution (Netzer 2009) and its evolution with redshift (Labita et al. 2009), as well as the luminosity function of AGNs as a function of redshift (e.g., Ueda et al. 2003). We can then combine all this information to calculate the density and evolution of the $\mathrm{Fe} \mathrm{K} \alpha$ line from accretion disks. In the following we concentrate solely on the $\mathrm{Fe} \mathrm{K} \alpha$ line that arises from the inner accretion disk, and neglect the contribution from any narrow component (e.g., Nandra 2006), or one that arises from reflection off a Compton-thick absorber (e.g. Murphy \& Yaqoob 2009). The following $\Lambda$-dominated cosmology is assumed (Spergel et al. 2003): $H_{0}=70 \mathrm{~km} \mathrm{~s}^{-1} \mathrm{Mpc}^{-1}, \Omega_{\Lambda}=0.7$, and $\Omega_{m}=0.3$. 


\section{Calculations}

The 2-10 keV X-ray luminosity function measured by Ueda et al. (2003) is used as the basis for the density and evolution of AGNs. We consider 261 X-ray luminosities between $10^{41.5}$ and $10^{48} \mathrm{erg} \mathrm{s}^{-1}$, and assume a bolometric correction of 50 to convert these to $L_{\text {bol }}$ (Vasudevan \& Fabian 2007). The black hole mass distribution at $z \approx 0.15$ measured by Netzer (2009) is converted into 28 mass bins (spanning $M_{\mathrm{BH}}=1.7 \times 10^{6} M_{\odot}$ to $9.1 \times 10^{8} M_{\odot}$ ) and normalized to sum to unity. Applying this distribution to each of the 261 luminosities yields 261 Eddington ratio distributions. At $z=0.15$ the minimum and maximum Eddington ratios are $\lambda=1.4 \times 10^{-4}$ (at a X-ray luminosity of $10^{41.5} \mathrm{erg} \mathrm{s}^{-1}$ ) and $2 \times 10^{5}$ (at a Xray luminosity of $10^{48} \mathrm{erg} \mathrm{s}^{-1}$ ), respectively. A constant density ionized reflection code is used to calculate the reflection continuum including the $\mathrm{Fe} \mathrm{K} \alpha$ line (Ross \& Fabian 1993; Ross et al. 1999; Ross \& Fabian 2005). In these models, the density of the accretion disk is taken to be $n_{\mathrm{H}}=10^{15} \mathrm{~cm}^{-3}$, and $F_{X}$ is changed in order to obtain the desired value of $\xi$. The observed relationships between $\xi, \Gamma, A_{\mathrm{Fe}}$ and $\lambda$ (Risaliti et al. 2009; Inoue et al. 2007; Netzer \& Trakhtenbrot 2007) are used to determine the input parameters for the the reflection code over a wide range of $\lambda$. The parameters are frozen at reasonable values 1 for very high or low values of $\lambda$. The relationships are:

$$
\begin{gathered}
\Gamma= \begin{cases}0.58(\log \lambda+1.0)+1.99 & -2.0 \leq \log \lambda \leq-0.035 \\
2.55 & \log \lambda>-0.035\end{cases} \\
A_{\mathrm{Fe}}= \begin{cases}\left(10^{0.7 \log \lambda+0.59}\right) / 0.6 & 0.01<\lambda \leq 2 \\
10.45 & \lambda>2\end{cases}
\end{gathered}
$$

and

$$
\log \xi= \begin{cases}1.0 & \log \lambda \leq-1 \\ 2.0(\log \lambda+1)+1 & -1<\log \lambda \leq 1.5 \\ 6.0 & \log \lambda>1.5\end{cases}
$$

The Fe K $\alpha$ equivalent width (EW) and line flux are measured for each $\lambda$ and then averaged over the $\lambda$-distribution to obtain mean values for that particular X-ray luminosity. The accretion disk is not expected to be optically thick to X-rays when $\lambda \leq 0.01$ (Narayan \& Yi

\footnotetext{
${ }^{1}$ The upper limit of $\Gamma=2.55$ was chosen for computational reasons, but, despite the rare example (e.g., Fabian et al. 2009), the vast majority of AGNs have photon indices less than this value (e.g., Risaliti et al. 2009).
} 
1995), and in those cases the Fe K $\alpha$ line EW and line flux are set to zerd2. These calculations are repeated for 40 different redshifts between $z=0.05$ and $z=2$, with the black hole mass distribution moving to larger masses as $z^{0.3}$ (Labita et al. 2009).

It is currently unknown where the X-ray source is located in relation to the accretion disk in AGNs, and so we consider two limiting cases for $R$. The first is to assume $R=1$, a commonly observed value in measurements of broad Fe lines (Nandra et al. 2007), for all calculations of the $\mathrm{Fe} \mathrm{K} \alpha \mathrm{EW}$ and line flux. This is equivalent to assuming the accretion disk subtends a solid angle of $\sim 2 \pi$ as viewed from the X-ray source. The illuminating power-law and reflection spectra are simply added together to form the $R=1$ spectra. The second case assumes that $R$ is inversely proportional to $\lambda$, which is a prediction of models of relativistic light bending in AGNs where the X-ray source is located on the axis of the central black hole (Miniutti \& Fabian 2004). The relationship between $R$ and $\lambda$ is determined from the Suzaku measurements of the Seyfert 1 galaxy MCG-6-30-15 that assumed a variable $R$ (Miniutti et al. 2007). A black hole mass of $4 \times 10^{6} \mathrm{M}_{\odot}$ (McHardy et al. 2005), and a 2-10 keV bolometric correction of 50 (Vasudevan \& Fabian 2007) was used to calculate $\lambda$ for the two states of MCG-6-30-15. A straight-line fit yielded:

$$
R= \begin{cases}8.0 & \lambda \leq 0.09 \\ -8.5 \lambda+8.8 & 0.09<\lambda \leq 0.92 \\ 1.0 & \lambda>0.92 .\end{cases}
$$

In both cases, the $\mathrm{Fe} \mathrm{K} \alpha$ equivalent widths and line fluxes are computed by integrating over the total spectrum from $6 \mathrm{keV}$ to $7.1 \mathrm{keV}$. For the EW calculation, the continuum underneath the line is estimated by fitting a straight line to the spectrum between those two energies. To calculate the line flux an emitting area from the disk had to be assumed. Following the line emission model of Nandra et al. (2007), we assumed that each line was emitted from 1.235-6 $r_{g}$ with a flat emissivity index, and from 6-400 $r_{g}$ with an emissivity index of -3 . Here $r_{g}=G M_{\mathrm{BH}} / c^{2}$ is the gravitational radius of a black hole with mass $M_{\mathrm{BH}}$, $G$ is the gravitational constant and $c$ is the speed of light.

\footnotetext{
${ }^{2}$ Observations of Galactic black hole binaries seem to indicate that inner edge of the disk is a function of $\lambda$ (e.g., Gierliński et al. 2008), even when $\lambda>0.01$, although other observations imply a more stable inner radius (e.g., Miller et al. 2006). If such an effect occurs in AGN disks, then the number density of relativistic lines from the lower $\lambda$ sources will be reduced. However, there is currently no observational evidence of such an effect in Seyfert galaxies or quasars.
} 


\section{Results}

At any $z$, multiplying the observed X-ray luminosity function by the slope of the computed luminosity-EW relationship results in the Fe K $\alpha$ EW analogy to the AGN luminosity function, which we call the Fe $\mathrm{K} \alpha \mathrm{EW}$ function. Figure 1 plots this function at four different redshifts. There are strong peaks in the $R=1$ function at EWs of $\sim 110 \mathrm{eV}$ and $\sim 300 \mathrm{eV}$ at all redshifts that correspond to strong neutral and ionized $\mathrm{Fe} \mathrm{K} \alpha$ lines, respectively. In contrast, the strongest peak in the light-bending model is at an EW of $\sim 500 \mathrm{eV}$ which corresponds to neutral line emission that is enhanced by strong reflection. The $R=1$ model indicates that $\mathrm{Fe} \mathrm{K} \alpha$ lines with $\mathrm{EWs}<110 \mathrm{eV}$ will dominate the space density at all redshifts, while the light-bending model predicts a significant space density of very strong $\mathrm{Fe} \mathrm{K} \alpha$ lines with EWs up to $\sim 500 \mathrm{eV}$.

Each panel in Fig. 1 also gives the average Fe $\mathrm{K} \alpha \mathrm{EW}$ at the specified redshift for the two different models. The average EW for the $R=1$ model is $\sim 50 \mathrm{eV}$ for $z<1$ and then rises to $\sim 70 \mathrm{eV}$ at higher redshifts. In the extreme light-bending model, the average $\mathrm{Fe} \mathrm{K} \alpha$ $\mathrm{EW}$ is $\sim 200 \mathrm{eV}$ over the entire redshift range.

Figure 1 also shows a clear difference in the expected density of relativistic Fe $\mathrm{K} \alpha$ with EWs $>100 \mathrm{eV}$ between the $R=1$ and the light-bending models. Although there exists up to a factor of 10 scatter in the observed relationships connecting $\Gamma, \xi, A_{\mathrm{Fe}}$ and $\lambda$ (Risaliti et al. 2009; Inoue et al. 2007; Netzer \& Trakhtenbrot 2007), this scatter could in no way explain the three order of magnitude difference between the $R=1$ and light-bending models. Moreover, the decrease in high EW Fe K $\alpha$ lines in the $R=1$ model is driven by the

observed X-ray luminosity function. A recent compilation by Nandra et al. (2007) found that the majority of broad Fe K $\alpha$ components in nearby Seyferts have EWs less than $100 \mathrm{eV}$, in agreement with the $\mathrm{Fe} \mathrm{K} \alpha$ function for $R=1$. At $z=0.05$, the light-bending model predicts a space density of AGNs with Fe K $\alpha$ EWs $>100 \mathrm{eV}$ that is $5 \times$ larger than the $R=1$ model (this ratio drops to $\approx 1$ at $z=1$ ); for $\mathrm{EWs}>200 \mathrm{eV}$ the space-density is $235 \times$ larger in the light-bending model (this ratio drops to $\sim 150$ at $z=1$ ). Observations indicate that such intense lines are rare (Nandra et al. 2007), despite the fact that they would be the easiest to detect. However, our calculations assumed only one disk-like reflector in an AGN, but this may be violated for AGNs in certain states (Fabian et al. 2002a) where multiple disk reflections can boost the observed EW. Such a scenario seems to be required to explain the very large Fe K $\alpha$ EW observed from 1H 0707-495 (Fabian et al. 2009).

The predicted sky density distribution of relativistic Fe K $\alpha$ lines in the range $z=0.05-$ 0.5 is shown in Figure 2. This plot dramatically illustrates that, in the $R=1$ scenario, AGNs with Fe $\mathrm{K} \alpha \mathrm{EWs}>200 \mathrm{eV}$ will be very rarely observed between $z=0.05$ and 0.5. On the other hand, if the X-ray source in AGNs is located above the black hole, as in the 
extreme light-bending model, then such large EWs would be common, with sky densities of $\sim 500 \mathrm{deg}^{-2}$. If this model was generally true, then a near flat distribution of EWs should be observed, while, in contrast, the majority of detected broad Fe K $\alpha$ lines have EWs $\leq 100 \mathrm{eV}$ (Nandra et al. 2007), in agreement with the $R=1$ curve. The bottom line is that, unless extreme light-bending is increasing the value of $R$, relativistic Fe K $\alpha$ lines with EWs $\gtrsim 300 \mathrm{eV}$ can only be produce by ionized accretion disks with large Fe abundances. Such a situation is most common when an AGN has a large Eddington ratio, and such AGNs are rare at $z<1$.

One can use these results to plan future Fe $\mathrm{K} \alpha$ line surveys performed by the proposed International X-ray Observatory (IXO). Figure 3 plots the predicted sky density of $\mathrm{Fe} \mathrm{K} \alpha$ lines greater than a certain line flux in AGNs between $z=0.05$ and 2 . The plot shows that both the $R=1$ scenario and the extreme light-bending model predict that a small number of very bright lines with fluxes $>10^{-12} \mathrm{erg} \mathrm{cm}^{-2} \mathrm{~s}^{-1}$. For reference, the Fe $\mathrm{K} \alpha$ line in the Seyfert 1 galaxy MCG-6-30-15 is one of the brightest known and has a line flux of $\sim 2 \times 10^{-12} \mathrm{erg} \mathrm{cm}^{-2} \mathrm{~s}^{-1}$ (Vaughan \& Edelson 2001). This plot predicts that there should only be $\sim 8$ AGNs over the entire sky with Fe K $\alpha$ lines that have fluxes equal to or greater than MCG-6-30-15, in agreement with the observed scarcity of such lines. At lower fluxes, the predictions of the two models diverge with the difference approaching 2 orders of magnitude at fluxes of $\sim 10^{-15} \mathrm{erg}^{-2} \mathrm{~s}^{-1}$. Such a difference will be easily distinguished by future surveys by $I X O$, but could be measured by a careful analysis of the XMM-Newton and Suzaku archives.

\section{Concluding Remarks}

This work has shown, for the first time, the cosmic density and EW distribution of relativistic Fe K $\alpha$ lines. Sensitive observations of these line profiles are vital as they allow measurements of fundamental parameters such as the spin of the central black hole. Our results validate the previous and current results from XMM-Newton and Suzaku that have shown that the majority of relativistic Fe K $\alpha$ lines have EWs $<100 \mathrm{eV}$ (Nandra et al. 2007). Now that models of the intrinsic line EW and flux distributions are available, detailed planning of future Fe K $\alpha$ surveys by IXO can be performed. The sensitivity of IXO will push spin measurements beyond the local universe and out to moderate redshifts, revolutionizing our understanding of the cosmic evolution of black holes.

Finally, we have shown that the extreme light-bending model predicts many more intense relativistic Fe K $\alpha$ lines than the $R=1$ model (Figs. 1 and 2), in disagreement with current observational constraints. This implies that, on average, the X-ray source in AGNs does not 
lie on the axis of the black hole where light-bending would be so extreme. It seems more likely that the X-ray source in average AGNs lies above the accretion disk at a radius of several $r_{g}$ from the black hole where light bending is less severe. Comparing data from future surveys of Fe $\mathrm{K} \alpha$ lines with plots such as Figure 3 should allow a measurement of the average radial displacement of the X-ray source. Of course, this conclusion does not preclude the possibility that extreme light-bending occurs in a few rare sources.

The author thanks J.M. Miller and C.S. Reynolds for comments and advice. This paper is dedicated to the memory of Don Ballantyne.

\section{REFERENCES}

Ballantyne, D. R., Fabian, A. C., \& Ross, R. R. 2002, MNRAS, 329, L67

Ballantyne, D. R., \& Ross, R. R. 2002, MNRAS, 332, 777

Brenneman, L. W., \& Reynolds, C. S. 2006, ApJ, 652, 1028

Fabian, A. C., Ballantyne, D. R., Merloni, A., Vaughan, S., Iwasawa, K., \& Boller, T. 2002a, MNRAS, 331, L35

Fabian, A. C., Rees, M. J., Stella, L., \& White, N. E. 1989, MNRAS, 238, 729

Fabian, A. C., et al. 2002b, MNRAS, 335, L1

-. 2009, Nature, 459, 540

George, I. M., \& Fabian, A. C. 1991, MNRAS, 249, 352

Gierliński, M., Done, C., \& Page, K. 2008, MNRAS, 388, 753

Grandi, P., Tagliaferri, G., Giommi, P., Barr, P., \& Palumbo, G. G. C. 1992, ApJS, 82, 93

Inoue, H., Terashima, Y., \& Ho, L. C. 2007, ApJ, 662, 860

Labita, M., Decarli, R., Treves, A., \& Falomo, R. 2009, MNRAS, 396, 1537

Laor, A. 1991, ApJ, 376, 90

McHardy, I. M., Gunn, K. F., Uttley, P., \& Goad, M. R. 2005, MNRAS, 359, 1469

Miller, J. M. 2007, ARA\&A, 45, 441 
Miller, J. M., Homan, J., Steeghs, D., Rupen, M., Hunstead, R. W., Wijnands, R., Charles, P. A., \& Fabian, A. C. 2006, ApJ, 653, 525

Miniutti, G., \& Fabian, A. C. 2004, MNRAS, 349, 1435

Miniutti, G., et al. 2007, PASJ, 59, 315

Murphy, K. D., \& Yaqoob, T. 2009, MNRAS, 397, 1549

Nandra, K. 2006, MNRAS, 368, L62

Nandra, K., O’Neill, P. M., George, I. M., \& Reeves, J. N. 2007, MNRAS, 382, 194

Nandra, K., \& Pounds, K. A. 1994, MNRAS, 268, 405

Nandra, K., Pounds, K. A., Stewart, G. C., Fabian, A. C., \& Rees, M. J. 1989, MNRAS, 236, 39P

Narayan, R., \& Yi, I. 1995, ApJ, 452, 710

Netzer, H. 2009, ApJ, 695, 793

Netzer, H., \& Trakhtenbrot, B. 2007, ApJ, 654, 754

Pounds, K. A., Nandra, K., Stewart, G. C., George, I. M., \& Fabian, A. C. 1990, Nature, 344,132

Reynolds, C. S., \& Fabian, A. C. 2008, ApJ, 675, 1048

Reynolds, C. S., \& Nowak, M. A. 2003, Phys. Rep., 377, 389

Risaliti, G., Young, M., \& Elvis, M. 2009, ApJ, 700, L6

Ross, R. R., \& Fabian, A. C. 1993, MNRAS, 261, 74

-. 2005, MNRAS, 358, 211

Ross, R. R., Fabian, A. C., \& Young, A. J. 1999, MNRAS, 306, 461

Spergel, D. N., et al. 2003, ApJS, 148, 175

Tanaka, Y., et al. 1995, Nature, 375, 659

Ueda, Y., Akiyama, M., Ohta, K., \& Miyaji, T. 2003, ApJ, 598, 886 
Uttley, P. 2007, in Astronomical Society of the Pacific Conference Series, Vol. 373, The Central Engine of Active Galactic Nuclei, ed. L. C. Ho \& J.-W. Wang, 149-+

Vasudevan, R. V., \& Fabian, A. C. 2007, MNRAS, 381, 1235

Vaughan, S., \& Edelson, R. 2001, ApJ, 548, 694

This preprint was prepared with the AAS LATEX macros v5.2. 


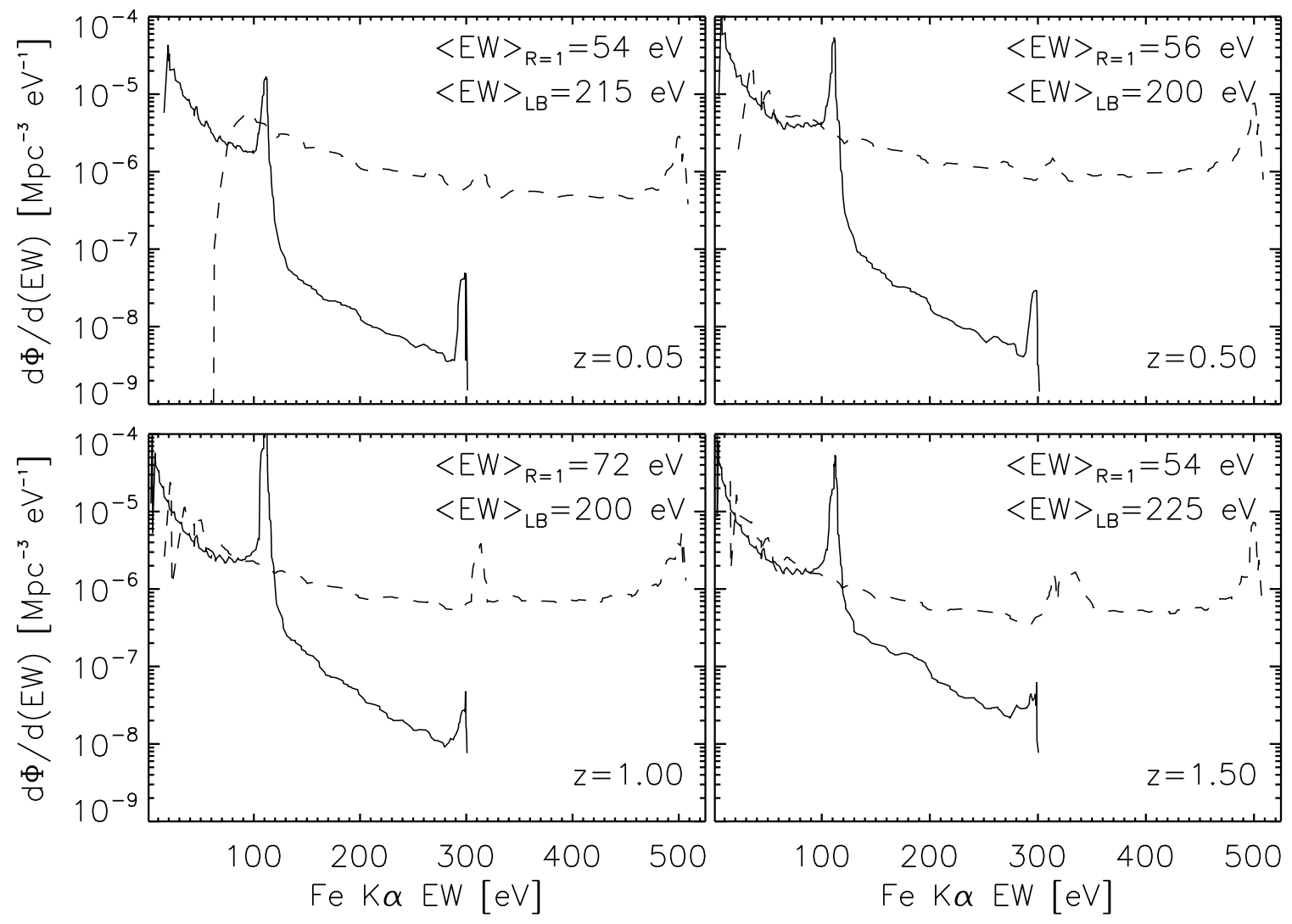

Fig. 1. - The rest-frame Fe K $\alpha$ equivalent width function at $z=0.05,0.50,1.0$ and 1.5. The solid line plots the function assuming a constant $R=1$, while the dashed line follows the strong light-bending prescription. The peaks in the curves indicate EWs that are most common at that redshift. For the $R=1$ model the peaks correspond to neutral Fe K $\alpha$ lines (at $\sim 110 \mathrm{eV}$ ) and ionized reflection (at $\sim 300 \mathrm{eV}$ ). In the light-bending model, the lines are always produced by neutral iron. The roll-over at low EWs in the light-bending model at $z=0.05$ corresponds to ionized $\mathrm{Fe} \mathrm{K} \alpha$ lines from luminous quasars (which have very low space density at that redshift). The average EWs for both models are also indicated in the plots. The curves have been smoothed for clarity. 


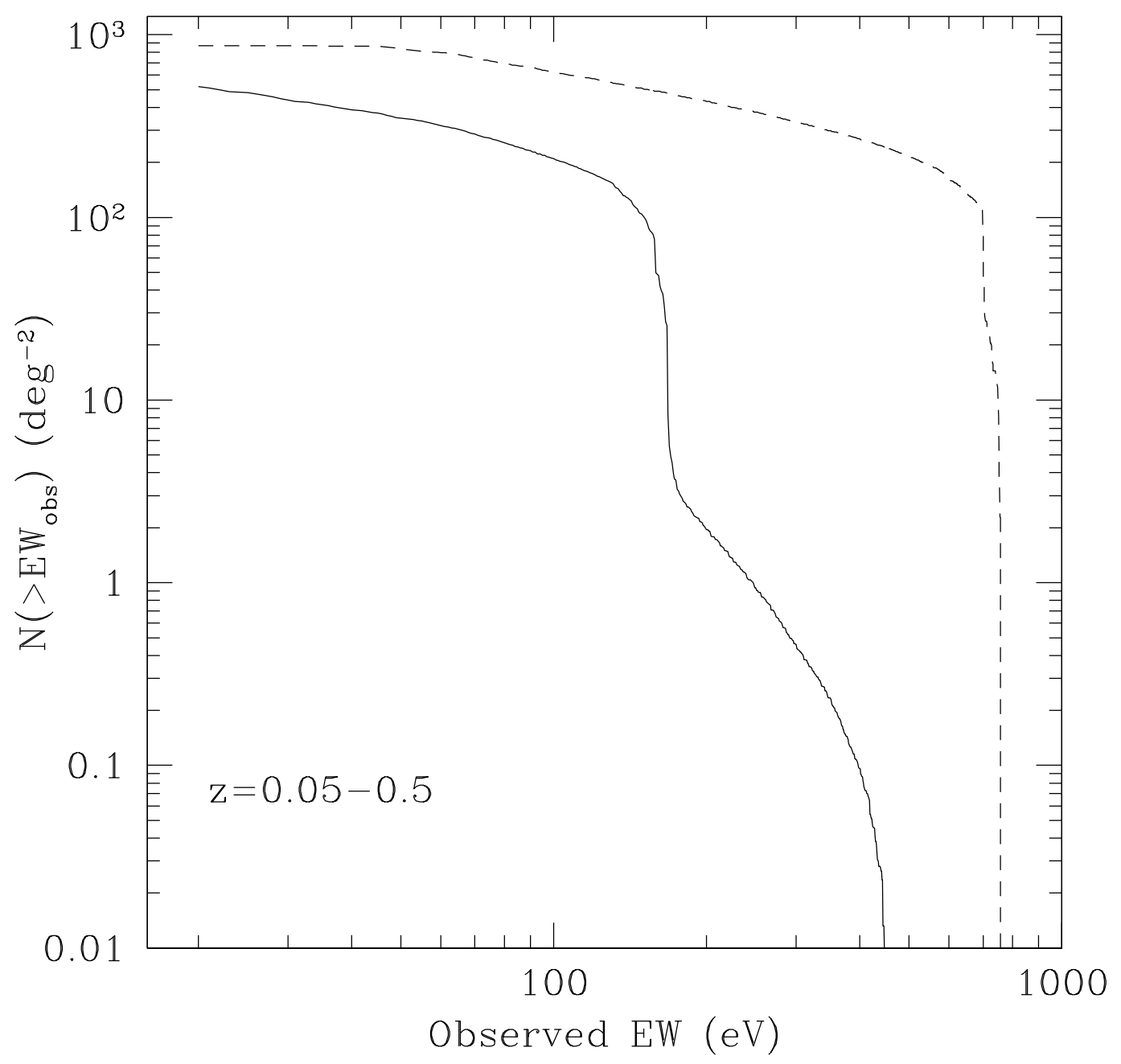

Fig. 2.- The expected sky density of relativistic Fe K $\alpha$ lines from AGNs in the range $z=$ 0.05- 0.5 with EWs greater than a certain observed value. The solid line plots the distribution from the models with a constant $R=1$, while the dashed line shows the predictions from the light-bending model where $R$ is inversely proportional to $\lambda$. The EWs in this plot are measured in the observed frame. 


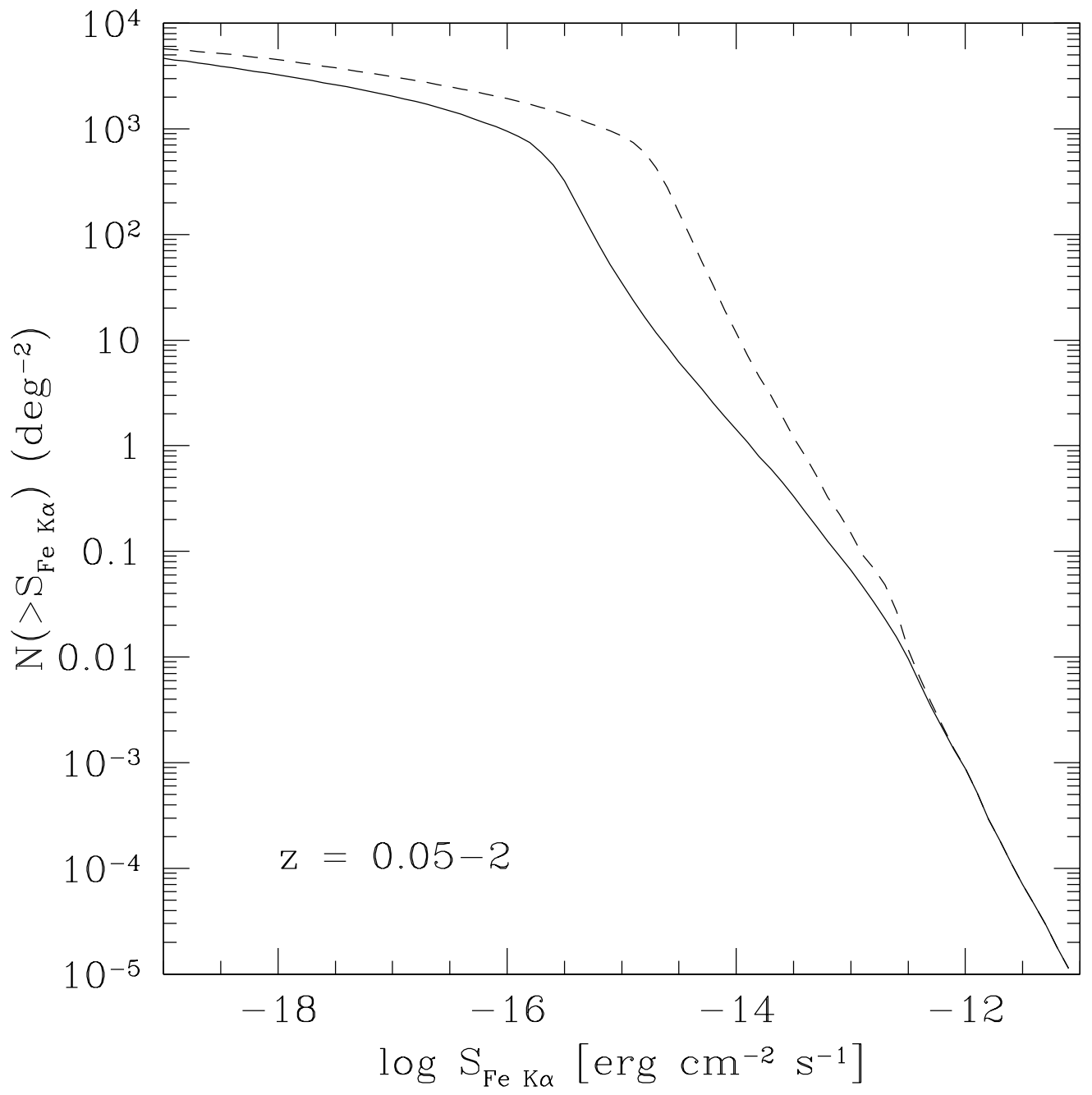

Fig. 3.- The expected sky density of relativistic Fe K $\alpha$ lines from AGNs in the range $z=0.05-2$ with line fluxes greater than a certain value. The solid line plots the distribution from the models with a constant $R=1$, while the dashed line shows the predictions from the light-bending model where $R$ is inversely proportional to $\lambda$. 